\title{
Developing the Geriatric Injury Documentation Tool (Geri-IDT) to Improve Documentation of Physical Findings in Injured Older Adults
}

\author{
Alexis Coulourides Kogan, $P h D^{7}$, Tony Rosen, $M D, M P H^{2}$, Adria Navarro, $P h D^{7}$, Diana Homeier, $\mathrm{MD}^{1,3}$, \\ Krithika Chennapan, $B S^{l}$, and Laura Mosqueda, $M D^{7}$
}

\begin{abstract}
'Department of Family Medicine and Geriatrics, Keck School of Medicine of USC, University of Southern California, Los Angeles, CA, USA;
${ }^{2}$ Department of Internal Medicine, Keck School of Medicine of USC, University of Southern California, Los Angeles, NY, USA; ${ }^{3}$, LAC+USC Geriatric Clinic and Adult Protection Team, Los Angeles, CA, USA.
\end{abstract}

BACKGROUND: Standardization in tools and documentation of child abuse and intimate partner violence have proven helpful in completely documenting injuries and suspected abuse among these populations. Similar tools do not yet exist for older adults and elder abuse.

OBJECTIVE: To (1) use insights from experts to develop a tool to assist clinicians in appropriately and completely documenting physical findings in injured older adults for potential future forensic investigation of abuse or neglect and (2) to assess the feasibility of incorporating this tool into clinical practice.

DESIGN: Two-phase, exploratory qualitative study. Phase 1: individual interviews with elder abuse experts from various specialties in medicine and criminal justice. Phase 2: focus groups with anticipated end users of the tool.

PARTICIPANTS: Phase 1 telephone-based key informant interviews were conducted with 11 elder abuse experts (2 detectives, 3 prosecutors, 1 forensic pathologist, 2 geriatricians, and 3 emergency medicine physicians). Phase 2 focus groups were conducted among emergency medicine $(n=10)$ and primary care $(n=8)$ providers.

APPROACH: Key informant interviews were conducted telephonically while the two focus groups were held inperson at an emergency medicine site in New York, NY, and a primary care site in Los Angeles, CA.

KEY RESULTS: Experts agreed that medical providers' documentation of geriatric injuries is usually inadequate for investigating alleged elder abuse/neglect. They highlighted elements needed for forensic investigation: initial appearance before treatment is initiated, complete head-to-toe evaluation, documentation of all injuries (even minor ones), and documentation of pertinent negatives. Several noted the value of photographs to supplement written documentation. End users identified practical challenges to utilizing a tool, including the burden of additional or parallel documentation in a busy clinical setting, and how to integrate it into existing electronic medical records.

CONCLUSION: A practical tool to improve medical documentation of geriatric injuries for potential forensic use

Electronic supplementary material The online version of this article (https://doi.org/10.1007/s11606-019-04844-8) contains supplementary material, which is available to authorized users.

Received April 12, 2018

Revised October 22, 2018

Accepted January 10, 2019

Published online February 13, 2019 would be valuable. Practical challenges to utilization must be overcome.

KEY WORDS: documentation tool; older adults; medical documentation; physical injury.

J Gen Intern Med 34(4):567-74

DOI: $10.1007 / \mathrm{s} 11606-019-04844-8$

๑) Society of General Internal Medicine 2019

\section{INTRODUCTION}

Health care providers who practice in emergency departments, outpatient offices, nursing homes, and hospitals commonly evaluate older adults with evidence of physical injury. For many of these patients, the injury may be linked to elder abuse or neglect. This elder mistreatment occurs commonly, with as many as $10 \%$ of older adults experiencing some form of abuse each year. ${ }^{1-3}$ However, the link between the injury and elder abuse is often not identified during the medical encounter. It may be weeks, months, or even years later that abuse is first suspected. An important challenge in the investigation and prosecution of these elder abuse cases is incomplete or inadequate documentation of injuries by the medical provider who initially assessed and treated them. In many cases, the quality of this documentation can significantly impact whether justice and protection can be achieved for a victimized older adult.

The importance of clinician documentation of injury-related findings in children for child abuse investigation is well-recognized, ${ }^{4,5}$ and research has shown that injury documentation in this population is often incomplete and inadequate. ${ }^{5-8}$ Reasons likely include clinicians' lack of training, time limitations, and the low immediate clinical significance of some injuries and physical findings. ${ }^{9}$ Additionally, potentially valuable ancillary strategies to enhance documentation of injuries such as photography are currently underutilized, ${ }^{9}, 10$ due to technical challenges and privacy concerns.

Tools to assist clinicians and improve and standardize documentation for future forensic investigation of child abuse and intimate partner violence have proven helpful in completely documenting injuries in children ${ }^{11,12}$ and younger adults. ${ }^{13,14}$ To our knowledge, similar tools do not yet exist for older adults. 
While the purpose of documentation tools for child abuse and intimate partner violence are to standardize, record, and formally report details of suspected abuse to law enforcement, several components may still be applicable for documenting any type of physical finding in older adult patients, whether abuse is suspected or not. Key components include the physical exam and body diagrams. The purpose of this study was to use insights from experts to inform the development of a universal tool to assist clinicians in appropriately and completely documenting physical findings in injured older adults for potential future forensic investigation of abuse or neglect and to assess the feasibility of incorporating this tool into clinical practice. This tool is intended to be used during medical encounters for all older adult patients that present with physical injury(ies) whether or not elder abuse is actually suspected.

\section{METHODS}

To achieve these aims, we conducted a two-phase exploratory, qualitative study. Phase 1: Tool development involved individual interviews with elder abuse experts from various specialties in medicine and criminal justice. Phase 2: Feasibility involved focus groups of anticipated end users of the tool, health care providers from primary care and emergency medicine settings. The Institutional Review Board of the University of [the University of Southern California] approved this study. The study report follows the Consolidated Criteria for Reporting Qualitative Studies Guidelines. ${ }^{15}$

\section{Development of Preliminary Draft of Tool Using Literature Review}

Before conducting interviews with experts, the research team developed a preliminary draft version of the proposed documentation tool. The research team, which has deep and diverse experience in elder mistreatment, family medicine, emergency medicine, and geriatrics/gerontology, conducted a comprehensive, non-systematic literature review (through January 2016) to inform development. This preliminary version incorporated previous research ${ }^{11,16-19}$ into a concise clinical document that included multi-view body diagrams. Review of existing clinical documentation tools for suspected child abuse and intimate partner violence was also an important piece that informed the development of the geriatric injury documentation tool. These tools typically involve structured forms to ensure complete and standardized documentation of the history and physical exam. We focused on the physical examination component of tools and noted several elements that were adapted and used for our initial design: instructions on how to optimally and completely describe various injuries, and use of a body diagram including close-ups of the face and genitalia to facilitate accurate injury location. The four-page tool (preliminary draft) consisted of a cover page (instructions, a table of common physical findings and important characteristics to document, and questions about mechanism and timeline of injury(ies) and who was present while history was reported) and three pages of body diagrams/traumagrams on which the health care provider may draw/provide details of findings ( 1 . front and back full body, 2. transverse full body, and 3 . multiple views of the head/face and neck (front, back, and sides), view of mouth, and view of male and female genitalia).

\section{Phase 1: Key Informant Interviews to Inform Tool Development}

We conducted key informant interviews via telephone with a purposive sample of elder abuse experts across the United States from multiple disciplines to inform development of the tool. We attempted to recruit participants with a wide range of medical and criminal justice experience and expertise, and potential participants were selected from the National Center on Elder Abuse (NCEA) coalition list. From June to September 2016, 15 elder abuse experts were identified and invited to participate as key informants. Eleven agreed to participate: six physicians (two geriatricians, three emergency medicine, one forensic pathologist) and five criminal justice representatives (two detectives, three prosecutors). The four individuals that did not participate were excluded because they either had changed jobs and new contact information was not available $(n=3)$ or had scheduling availability incompatible with interview facilitators $(n=1)$.

Interviews with each individual participant were conducted telephonically and were facilitated by two members of the researcher team: (1) a physician co-primary investigator (LM or TR) and (2) a postdoctoral researcher (ACK). Interviews were guided by a five-item semi-structured research protocol (see Appendix A) that was developed by the study team and tested for content and face validity by two geriatricians (LM and DH) and an emergency medicine physician (TR) with extensive clinical and research experience in elder mistreatment. The research protocol followed an inductive approach - seeing how individual observations turn into patterns, then broader themes - to elicit experts' input on the content and format of crucial items to include in a clinical tool to document physical findings in older adult patients. Participants were not given the questions prior to their interview. They were, however, electronically given a draft documentation tool at the end of the interview and specifically asked to provide feedback on the tool (final interview question). Participants received an informed consent document prior to the interview, and verbal consent was obtained at the beginning of each interview. Interviews were audio-recorded and transcribed verbatim. Data saturation was achieved after seven interviews. Four additional interviews were conducted for thoroughness and equal representation across the targeted medical and law enforcement fields.

\section{Analysis}

Transcripts from each of the 11 interviews were analyzed using a grounded theory approach ${ }^{20,21}$ to identify and code themes that arose from the data. Three researchers (ACK, AN, 
and $\mathrm{KC}$ ) open coded the transcripts by noting ideas and relationships between concepts and categories and identified initial themes. Next, working independently, they identified key phrases and passages that expressed distinct themes - ideas, descriptions, narratives, attitudes, beliefs, and values - and generated preliminary code lists. ${ }^{22}$ After the initial cycle of open coding, ${ }^{23}$ the authors held a consensus meeting and were able to discuss, sort, and refine the coding scheme, collapsing codes with similar meanings into distinct codes and sub-codes. The authors jointly reviewed the revised codes, and then all three independently recoded the transcripts using these new codes. The researchers then came together again to reconcile remaining coding differences to achieve greater than $90 \%$ consensus.

Based on this analysis of the feedback obtained in the key informant interviews, the study team revised the documentation tool during several team meetings and follow-up email conversations. This new, second draft (preliminary draft-revised) was used in the focus group study feasibility assessment phase.

\section{Phase 2: Focus Groups}

Two focus groups were held in September-October 2016 at an emergency medicine site in New York, NY, and a primary care site in Los Angeles, CA. The focus groups took place during a regularly-scheduled clinical team meeting at each site. Focus groups were facilitated by study personnel (NY: ACK and TR; CA: DH and ACK), verbal consent was obtained, and participants filled out a brief demographic questionnaire at the beginning of the focus group. Discussions were guided by a three-item semi-structured research protocol that was developed by the study team and tested for content and face validity by validity by a geriatrician geriatrician (LM) and an emergency medicine (TR) physician on the study team who each have extensive clinical and research experience in elder mistreatment. The research protocol followed an inductive approach to elicit clinicians' input on the feasibility of incorporating the documentation tool into their regular clinical practice for all older adult patients - regardless of whether elder abuse is suspected or not-as well as suggestions to improve its content and clarity. Participants were not given the questions prior to their interview, but were each given a draft documentation tool (preliminary draft—revised) to review during the focus group and a brief demographics questionnaire. Both focus groups were audio-recorded and transcribed verbatim.

Descriptive statistics were used to describe the sample of focus group participants using SPSS version 22. The transcripts were analyzed using grounded theory with the same team members and an identical approach to that described above for examination of key informant interviews. Based on the results of these focus groups, the research team made additional revisions and finalized the documentation tool during several meetings and follow-up e-mail conversations. Lastly, the final draft was vetted by focus group participants at both the emergency medicine and and primary care sites.

\section{RESULTS}

\section{Key Informant Interviews}

From June to September 2016, 11 elder abuse experts from across the United States participated in key informant interviews, each lasting between 21 and $57 \mathrm{~min}$. Qualitative analysis of interview transcripts led to identification of three broad, independent themes with subthemes: (1) current documentation practices, (2) the draft documentation guide, and (3) practical challenges. All experts agreed that medical providers' documentation of geriatric injuries is usually inadequate for purposes of investigating alleged elder abuse/neglect and that developing a tool would be valuable. They highlighted several elements critical for forensic investigation that should be included: initial appearance before treatment is initiated, complete head-to-toe evaluation, documentation of all injuries (even minor ones), and documentation of pertinent negatives. Respondents also validated the inclusion of elements in the original draft such as the types of characteristics to document and the types of injuries. Suggested additions included petechiae and pressure sores/ injuries as important and distinct types of injuries that should be added to the tool. Several experts also noted the value of photographs to supplement written documentation. Informants identified practical challenges to utilizing a tool for providers, including the burden of additional or parallel documentation in a busy clinical setting and how to integrate it into existing electronic medical records. Respondents from law enforcement discussed the idea that the value the geriatric injury documentation tool would add to a patient's medical encounter and record would far outweigh any time and administrative burden it might impose. Further details of qualitative findings are shown in Table 1.

\section{Focus Groups}

During the end of September-early October 2016, two focus groups were held with emergency medicine $(n=10)$ and primary care $(n=8)$ providers in New York and Los Angeles, respectively (demographic characteristics of participants are shown in Table 2). Focus groups lasted between 37 and $45 \mathrm{~min}$. Qualitative analysis of focus group transcripts led to identification of four broad, independent themes with subthemes: (1) tool edits, (2) utility, (3) multidisciplinary collaboration, and (4) challenges with incorporation into usual practice. Participants in both groups liked the tool and suggested several minor edits that pertained to formatting (e.g., item order and fitting the tool onto one front-and-back sheet of paper) and content (e.g., adding an item about whether alcohol or illicit drugs were involved). The body diagrams were perceived as especially helpful, and participants appreciated their inclusion. All agreed that the tool would potentially be useful to increase providers' completeness, detail, and accuracy in written documentation. Participants reported that, after discussion of the tool, they planned to review and change their own documentation practices. In both groups, there were robust 
Table 1 Perceptions of Documenting Physical Findings on Older Adult Patients

\begin{tabular}{lllcl}
\hline \hline Theme & $\begin{array}{l}\text { Participant } \\
\text { no. }\end{array}$ & Quote & Expertise
\end{tabular}

1. Current documentation practices

107 "I will say in general I think, there has been a sort of gradual decay in the quality of physician documentation which is probably related to copying and pasting and templating with electronic medical records and my impression is physical exams [chart section] are worse and worse and worse."

110 "I want to know: Do these medical findings jibe with what he's [perpetrator] saying and if everything is not documented, then I am not going to know." "I think as a whole profession, physicians, I think we do a really bad job with any sort of specificity with our physical exam in terms of skin and soft tissue injuries like this. So, I think that we probably just do not add enough detail in general."

2. The documentation guide

Subtheme 1. Key additions/missing content: physical exam

110 "I think that it's also important to document any other smaller injuries. A lot of times what I find in medical records is they'll document the bigger injuries, the ones that need to be treated....and we have found over history-whether it's elder abuse or not elder abuse-are not documented, but those injuries can make the difference between something looking like a fall or an accident versus multiple hits or grabbings."

102

"Well, I think the physical findings start at the time that the ED doors open or the first time that maybe they are seeing their general practitioner."

Subtheme 2. Key additions/missing content: context characteristics

103 "... and then not just sort of noting that there is pain but describing exactly how it's evident to the physician that they are [patient] in pain.... What we are proving in these cases almost always is what would have been evident to a lay person, caregiver."

“...If I think more broadly, it's like evidence of a wound infection-or you know, an infectionthat is clearly limb or life threatening and... for which the time course cannot possibly have been within the last one to two hours...I think where it's like clearly...the presentation suggests that there has been more than 24-hour delay in care...it's important relevant information to document."

"I wish that there was more in the cognitive screening area of things because we are seeing that along with the physical abuse. But we are also seeing the neglect in the area where people are declining cognitively, which also makes them vulnerable. Also if they are declining cognitively they also may be more work for somebody, they may be becoming combative, and they may require a higher level of care...”

Subtheme 3. Photographic documentation

101 "...The more important things is that something is documented and photographed...people should not get intimidated about picking the right word [abrasion vs. laceration vs. skin tear]. The important thing is that it's documented."

109 "...I'd rather see a picture and then try to describe it."

3. Practical challenges

Subtheme 1. Physician-level challenges

"I think there's always going to be that tendency for people rushing and probably not documenting and you know, I am sure I am guilty of this too, and not being as precise as you would want."

"...Physicians were taught when you write a physical exam, we say 'crackles in bilateral lung bases.' We do not say, 'crackles in bilateral lung bases suggest pulmonary edema,' we just describe them and then in another section of the note-let us say in the assessment and plan-we might say, 'physical exam suggests pulmonary edema.' So I wonder to what extent you want people physicians' to interpret their findings...this sort of runs up a little bit against the culture of medicine and how people describe findings...but if we use child abuse as a paradigm, we unequivocally ask physicians to do that kind of interpretation or at least to begin that type of interpretation as part of their evaluation."

\section{Subtheme 2. System-level challenges}

111

105 "[in regards to using photography in relation to the EMR] we are not using it in the emergency department. We do have medical photography so that if somebody comes in with let us say
decubiti...medical photography will always come and photograph that to document...they are more like Monday to Friday...I do not believe this photography is included in the medical record. To be honest, I have not seen, since switching our electronic medical record, I have not seen any photos that are part of a record."

"Our emergency department and health system uses the EPIC electronic coding system and that has a system already where you can download an app on any smartphone that securely connects you to the Epic chart and it [photos] will go right into the patient's chart...I would say that the majority of people either have that capability or there is someone on shift with them and they are like, 'Can I borrow your phone'..."
Emergency medicine physician

Prosecutor

Geriatrician

Prosecutor

Detective

Prosecutor

Emergency medicine physician

Detective

Forensic scientist

Prosecutor

Geriatrician

Emergency medicine physician

Emergency medicine physician

Emergency medicine physician discussions about incorporating the documentation tool into electronic medical records (EMRs). Several participants emphasized that this was critical, as it was the ideal format for integrating the tool into routine practice. Participants reported concern about the time involved in using the documentation tool for all older adult patients due to high patient volume, high number of physical findings on older adult patients, and uncertainty over injury versus non-injury physical findings. They acknowledged that some of these challenges could be mediated by including the documentation tool in the EMR. Opinions were mixed about whether other disciplines, such as nursing and social work, should be tasked with completing the 
Table 2 Focus Group Participant Characteristics $(n=18)$

\begin{tabular}{|c|c|c|c|}
\hline & $\begin{array}{l}\text { Emergency } \\
\text { department }\end{array}$ & $\begin{array}{l}\text { Primary } \\
\text { care }\end{array}$ & Overall \\
\hline & $n=10$ & $n=8$ & $n=18$ \\
\hline & \multicolumn{3}{|l|}{ Frequency (\%) } \\
\hline \multicolumn{4}{|l|}{ Gender } \\
\hline $\begin{array}{l}\text { Male } \\
\text { Female }\end{array}$ & $\begin{array}{l}6(60.0) \\
4(40.0)\end{array}$ & $\begin{array}{l}1(12.5) \\
7(87.5)\end{array}$ & $\begin{array}{l}7(38.9) \\
11 \\
(61.0)\end{array}$ \\
\hline \multicolumn{4}{|l|}{ Ethnicity } \\
\hline $\begin{array}{l}\text { Hispanic or Latino } \\
\text { Non-Hispanic or }\end{array}$ & $\begin{array}{l}0(0.0) \\
10(100.0)\end{array}$ & $\begin{array}{l}1(12.5) \\
7(87.5)\end{array}$ & $\begin{array}{l}1(5.6) \\
17 \\
(94.4)\end{array}$ \\
\hline \multicolumn{4}{|l|}{ Race } \\
\hline $\begin{array}{l}\text { Native American/ } \\
\text { Alaskan Native }\end{array}$ & $0(0.0)$ & $0(0.0)$ & $0(0.0)$ \\
\hline $\begin{array}{l}\text { Asian } \\
\text { Black or African }\end{array}$ & $\begin{array}{l}3(30.0) \\
0(0.0)\end{array}$ & $\begin{array}{l}0(0.0) \\
0(0.0)\end{array}$ & $\begin{array}{l}3(16.7) \\
0(0.0)\end{array}$ \\
\hline \multicolumn{4}{|l|}{ American } \\
\hline $\begin{array}{l}\text { Native Hawaiian or } \\
\text { other Pacific Islander }\end{array}$ & $0(0.0)$ & $1(12.5)$ & $1(5.6)$ \\
\hline White & $7(70.0)$ & $6(75.0)$ & $\begin{array}{l}13 \\
(72.2)\end{array}$ \\
\hline \multicolumn{4}{|l|}{ Age group } \\
\hline 39 years or younger & $3(30.0)$ & $3(37.5)$ & $6(33.3) r y-1)$ \\
\hline $40-49$ years & $3(30.0)$ & $3(37.5)$ & $6(33.3)$ \\
\hline $50-59$ years & $2(20.0)$ & $2(25.0)$ & $4(22.2)$ \\
\hline \multirow{2}{*}{\multicolumn{4}{|c|}{ Role }} \\
\hline & & & \\
\hline Physician (MD) & $9(90.0)$ & $6(75.0)$ & 15 \\
\hline $\begin{array}{l}\text { Physician assistant } \\
\text { (PA) }\end{array}$ & $1(10.0)$ & $2(25.0)$ & $3(16.7)$ \\
\hline
\end{tabular}

†Participant self-reported as "Black and White"

documentation tool as a way to reduce burden on the physician and save time. Additionally, focus group participants discussed the potential value of photography to supplement a written note; however, some acknowledged the methodological considerations and privacy challenges clinicians currently face. Further details of qualitative findings are shown in Table 3.

\section{DISCUSSION}

This study aimed to use insights from experts and frontline clinicians to inform the development of a tool to assist clinicians in appropriately and completely documenting physical findings in all injured older adults for potential future forensic investigation of abuse or neglect as well as the feasibility of implementing such a tool. To our knowledge, no other such tools exist, and, in designing this tool, our study is the first to explore expert perspectives on clinical documentation of physical injuries and wounds in older adult patients. The final version of the tool is available as Appendix B. We have named it Geri-IDT (Geriatric Injury Documentation Tool).

Our findings confirm the inadequacy of current clinical documentation for older adult patients for forensic purposes and the potential utility of a tool to guide and standardize it. Experts have identified critical elements to include and, in accordance with previous research, ${ }^{24}$ highlighted the value of photography. ${ }^{11}$ Not surprisingly, elements highlighted by our proposed tool are also found in existing, standardized tools for documenting child abuse and intimate partner violence that are already widely used in medical settings across the United States. Critical elements reported by our participants and found in existing documentation forms for child abuse and intimate partner violence include gathering history of the assault/injury/physical finding from both the victim and family separately, focus on the mechanism of injury, detailed description of exam findings, and use of a body diagram to depict findings as well as a recommendation to take photographs when possible. ${ }^{11}$

Participants also suggested several elements for the draft tool that were not included in the final version. These elements included documentation of the patient's level of cognition during the medical encounter, patient's emotional status, and purposeful documentation (i.e., physician editorialization in the EMR written note). Each of these suggestions was not included in the final Geri-IDT because they fall beyond the scope of the tool which focuses on physical injuries. However, these elements are very important details that may be better suited to be documented in the Assessment and Plan section of the EMR as opposed to the Physical Exam where the Geri-IDT would likely reside. Current practice for documenting all types of unequivocally suspected abuse cases (child, intimate partner, and elder) already finds physicians editorializing in the Assessment and $\mathrm{Plan}^{25}$ in addition to filing a formal report. Therefore, it may be beneficial to provide additional training for clinicians to be mindful about presentations, injuries, and patterns ${ }^{25}$ so that they may expound on documented findings from the physical exam in the Assessment and Plan when applicable. The potential value of improved geriatric injury documentation is enhanced by emerging research suggesting that important clinical differences may exist between abuserelated injuries and those that occur unintentionally. ${ }^{26-31}$ Therefore, good documentation for geriatric injuries may not only impact whether justice and protection can be achieved for a victimized older adult, but it can also enhance medical care and help to protect caregivers from unfounded suspicion. Further research is warranted in this area; however, in the field of geriatric medicine where patients often have physical findings on their skin (i.e., bruising and discoloration related to medications), it seems more important to err on the side of documenting everything rather than nothing. A comprehensive document such as the Geri-IDT can serve as a model for appropriate documentation for physical injuries in older adult patients and highlight the value of standardizing how findings are documented for all patients. Further testing of the GeriIDT is needed to examine its impact on care quality and improved documentation. The idea that photography can bring added value to clinical documentation of physical findings in older adult patients was also discussed as it relates to supplementing written documentation. However, previous research has identified several challenges with medical 
Table 3 Perceptions of the Geriatric Injury Documentation Tool (Geri-IDT)

\begin{tabular}{llll}
\hline \hline $\begin{array}{l}\text { Theme/ } \\
\text { subtheme }\end{array}$ & $\begin{array}{l}\text { Participant } \\
\text { no. }\end{array}$ & Quote & Setting
\end{tabular}

1. Tool edits 105

203

207

2. Utility

102

201

2a. Electronic health records

106

2b. Photography

\section{2c. Quality improvement}

211

3. Multidisciplinary collaboration 3a. Time

3b. Feasibility

102 and 105

4. Incorporation into usual practice 4a. Challenges

104

4b. Timing/workflow
"I would have it all on one page [front and back["

"This 'presence of tenderness': so I think while all this other stuff here is good, I would like to put that one on top .... The things we are less likely to do as clinicians should probably go higher on the list." "...I do not see any questions in terms of documentation about the use of alcohol or substances...This is very important."

"I think it's nice in that it kind of prompts you to be thorough and not miss things and then allow you to, like, easily document, you know, the nature of it, and...to track it over time. Even if it's a new patient, it kind of forces you to think about this stuff that maybe you would have missed otherwise. So to me it's kind of a nice guide."

"I think this is definitely something I would use. I think the, having the diagrams is really helpful because I think part of our... challenge is always accurately and succinctly enough describing the location of the injury and exactly where it is, and also potentially, how it looks especially if...it does not follow an exactly spherical pattern or whatnot. So I think this is really helpful in terms of having the free writing and the free drawing capabilities."

“...An electronic version [of the tool] with a dropdown option for marking would be the quickest way...I think it's great for what we want to document."

"So I think that if this assimilated into our practice, it would need to be in the electronic record and something that we could import into the physical exam."

"If we had a camera attached to the electronic health record and you just...took a picture, and then it came right in there [electronic health record] and you could type, 'This is a four by three centimeter...' and like it could all just be incorporated, I would use that."

"...I mean we can talk about smart pens and drawings but a picture is worth, you know, a thousand words... We may show five different people a picture and they are going to describe it differently. But if I showed five different people a rash, they still would describe it differently, even if they saw it in person. You know?"

"If the purpose is to capture as much details about as many patients as possible, it's going to be very, very helpful. If the objective is to make sure you do not miss anybody and that everyone is documenting exactly the same fashion, it's going to present several problems; depending on how busy the emergency department is...I think the details, the characteristics to document are so detailed that if you can capture all of these things when they are relevant, that would be great...It's better than freestyle because freestyle is they document whatever the hell comes into their head, which is never going to be the same. And I think the success of this will depend on how few injuries there are."

"One of the things that I think is so nice about this [documentation tool], and perhaps I am being particularly retrograde today, but I think there's a lost art in description when you document and when you even present. And I think that I see it, you know, in the residents...But I find that a lot of people do not do a thorough job of documenting what it is that they see and what's frankly important, both in terms of, you know, information passing and what really represents an elegant note both from a medical legal standpoint and, you know, and just from a quality standpoint. So anything that can improve that..."

"I think the real, very practical challenge with primary care is, you, know, we got to just take care of what we can in the time that we have."

"I think a multidisciplinary form would work as well because nursing's often doing this-the cupid eyethey're doing a lot of things that I may not be addressing because, you know, of other things I am doing. So making this not maybe a physician component but a multidisciplinary form where findings can be reported by anybody."

"...Would it be helpful or could we even do this... where the documentation tool could be used by the nurse that sees your patient first?"

"Yeah, that's what I was thinking. I would also wonder what the patient would feel like having someone examine their skin before they have seen their doctor...I just feel the nurses would be very uncomfortable doing that as a routine thing that's part of...because you would have to take off clothing in order to do [so], if you really wanted to find something."

"I think a dedicated practitioner, and it could be an NP, a PA, a nurse, physician, but probably not a physician, who evaluates every patient and does not have to be-I mean, that may not be about elderly people you believe... who were traumatized but every trauma patient that comes in, young, old. Solid consistency and that's their sole focus and their sole job, I think the documentation will be much, much better...like the Victim Intervention group."

"I would say, honestly, I see things on older patients that I have no idea if it's an injury or not because they have such, you know, they'll often have things on their arms. And I try to, you know, look for patterns but...there's often a lot [of physical findings]."

"...So if someone who has a couple injuries: yeah, I'll take the time and do everything that's there, to list every item. If they have more than two or three, you are going to lose the details of the individual lesions that are there... anything that you can do to enhance the ease, whether it's a scribe, whether it's a really well-designed EHR that then has to be compatible with all the EHRs that other hospitals are using...The information is critical, obviously. But how to do it without keeping the other 30 thousand patients in the waiting room waiting longer...”
$\mathrm{PC}$

ED

ED

PC

PC

PC

PC

PC

PC 


\begin{tabular}{lll}
\hline \hline $\begin{array}{l}\text { Theme/ } \\
\text { subtheme }\end{array}$ & $\begin{array}{l}\text { Participant } \\
\text { no. }\end{array}$ & Quote \\
\hline 103 & $\begin{array}{l}\text { "But I think if you make it like, 'This is just how we are documenting on any patient who comes in... PC } \\
\text { with a skin finding or something, and there's a form we can pop open [in the EHR] and document it } \\
\text { and then pop it back down and it is part of our charting for what we are doing for any patient who } \\
\text { comes in, then I think it works into the workflow." } \\
\text { [What would be the likelihood of using the tool to document any physical finding on your older adult PC } \\
\text { patients? "Very good on the first three patients of the day, and then almost nil after that." }\end{array}$ \\
\hline
\end{tabular}

$P C$ primary care, ED emergency department

photography, particularly when using personal mobile devices such as smart phones and tablets, including privacy concerns, $^{32,33}$ safe storage, ${ }^{32,34,35}$ and photo quality (e.g., methods for taking photos, location clearly depicted, size/ scale clearly portrayed). ${ }^{9,}{ }^{35}$ Although research in this area is currently bourgeoning, much of this work has focused on the use of personal mobile devices for telemedicine (triage, consultation, and diagnosis) ${ }^{36-38}$ and remote wound monitoring. ${ }^{39,}{ }^{40}$ Less work is focused on using photography to increase accuracy and detail of documentation of physical findings, though this has been recognized in the field of dermatology. ${ }^{41}$ Continued work in this area will be valuable to inform how photography using mobile devices can be effectively incorporated into medical encounters and contribute to improved medical documentation.

Participants also highlighted several practical challenges for clinicians incorporating a paper-based documentation tool into existing workflow in busy clinical settings including time pressures and additional or parallel documentation. A potential solution is to integrate the tool into the EMRs that are being used by an increasing number of health care providers. This would make it easier for providers to document information recommended by the tool, and prompt them to add anything that was missed. Additionally, many EMRs now include electronic versions of body diagrams that may be easily used to improve detail and accuracy for physical findings. Thus, incorporating this tool into EMRs is an important future step to ensure its use for all older adult patients. Though challenging given the diversity of existing EMRs, ${ }^{42}$ a reasonable approach would focus initially on those with the largest penetration. Additional testing would be necessary, of course, to optimize the Geri-IDT format for integration into various EMRs.

Next steps in Geri-IDT development involve pilot testing the paper documentation tool in primary care and emergency medicine settings and measuring key factors related to the tool's implementation, usability, acceptability, and impact on medical documentation as well as exploring potential integration into EMRs.

\section{Limitations}

This qualitative study has several limitations. We relied on a small purposive sample of experts working in the area of elder mistreatment, and although a great effort was made to recruit participants from across the country, results may not be generalizable beyond our sample. In this study, we approached poor physician documentation as a medical-legal issue and only included professional from these fields. However, not including the perspectives of social workers, geropsychologists, and adult protective service (APS) workers, among others, may limit study findings. Additionally, purposive recruitment of eligible subjects may have resulted in a study sample that could have been positively biased toward the importance of developing a clinical tool to document physical findings in older adult patients. While we recognize that a positive bias for documentation, even in the absence of suspected abuse, may introduce various burdens on clinicians, we believe that the time required to complete the Geri-IDT is time well spent. In the field of elder mistreatment where the link between injury and abuse often takes weeks, months, or even years to identify, complete documentation and a paper trail are invaluable.

\section{Practice Implications}

Using child abuse and intimate partner violence as a paradigm, creating and implementing a tool to document physical findings in older adult patients has the potential to improve clinical documentation and medical care for this patient population. Additionally, utilizing a simple tool for documentation will be helpful in any circumstance, whether related to abuse or not. Good documentation is as important for avoiding unwarranted accusations as it is for identification of suspected abuse. In a field that has been plagued by poor and inadequate documentation, any additional burden and time commitment associated with incorporating the Geri-ITD into medical encounters with older adult patients may be outweighed by the potential value it brings. We have developed the Geri-IDT using a literature review and a rigorous two-phase qualitative approach. We are currently working to test its acceptance and impact and to consider how to optimally incorporate it into EMRs.

Acknowledgements: The authors would like to thank all of the key informant interviewees and focus group participants that generously gave their time.

This study was funded by the Department of Justice (DOJ) and in part by the National Institute on Aging of the National Institutes of Health under award numbers 5 K99AG052647-02 and 4 ROOAG052647-03 for ACK's time. The content is solely the responsibility of the authors and does not necessarily represent the official views of the Department of Justice or National Institutes of Health. 
This study was presented as a poster at the American Geriatrics Society (AGS) annual meeting in San Antonio, TX, in May 2017.

Corresponding Author: Alexis Coulourides Kogan, $\mathrm{PhD}$; Department of Family Medicine and Geriatrics, Keck School of Medicine of USC, University of Southern California, Los Angeles, CA, USA (e-mail: acoulour@usc.edu).

\section{Compliance with Ethical Standards:}

Conflict of Interest: The authors declare that they do not have a conflict of interest.

Publisher's Note: Springer Nature remains neutral with regard to jurisdictional claims in published maps and institutional affiliations.

\section{REFERENCES}

1. Lifespan of Greater Rochester Inc., Weill Cornell Medical Center of Cornell University, New York City Department for the Aging. Under the radar: New York state elder abuse prevalence study: self-reported prevalence and documented case surveys. 2012. http://www.ocfs.state.ny.us/main/reports/Under\%20the $\% 20$ Radar $\% 2005 \% 2012 \% 2011 \% 20$ final\%20report. pdf. Accessed 12 December 2018.

2. Acierno R, Hernandez MA, Amstadter AB, et al. Prevalence and correlates of emotional, physical, sexual, and financial abuse and potential neglect in the united states: the national elder mistreatment study. Am J Public Health. 2010;100:292-7.

3. Lachs MS, Pillemer KA. Elder abuse. N Engl J Med. 2015;373:1947-56.

4. Dubowitz H, Bross DC. The pediatrician's documentation of child maltreatment. Am J Dis Child. 1992;146:596-9.

5. Jackson AM, Rucker A, Hinds T, Wright JL. Let the record speak: medicolegal documentation in cases of child maltreatment. Clin Pediatr Emerg Med. 2006;7:181-5.

6. Boyce MC, Melhorn KJ, Vargo G. Pediatric trauma documentation: adequacy for assessment of child abuse. Arch Pediatr Adolesc Med. 1996; 150:730-2.

7. Guenther E, Olsen C, Keenan H, Newberry C, Dean JM, Olson LM. Randomized prospective study to evaluate child abuse documentation in the emergency department. Acad Emerg Med. 2009;16:249-57.

8. Limbos MA, Berkowitz CD. Documentation of child physical abuse: how far have we come? Pediatrics. 1998;102:53-8.

9. Bloemen EM, Rosen T, Cline Schiroo JA, et al. Photographing injuries in the acute care setting: development and evaluation of a standardized protocol for research, forensics, and clinical practice. Acad Emerg Med. 2016;23:653-9.

10. Pasqualone GA. Forensic RNs as photographers: documentation in the ED. J Psychosoc Nurs Ment Health Serv. 1996;34:47-51.

11. Bar-on ME, Zanga JR. Child abuse: a model for the use of structured clinical forms. Pediatrics. 1996;98:429-33.

12. Newton AS, Zou B, Hamm MP, et al. Improving child protection in the emergency department: a systematic review of professional interventions for health care providers. Acad Emerg Med. 2010;17:117-25.

13. Ansari S, Boyle A. Emergency department-based interventions for women suffering domestic abuse: a critical literature review. Eur J Emerg Med. 2017;24:13-8

14. Ritchie M, Nelson K, Wills R, Jones L. Does training and documentation improve emergency department assessments of domestic violence victims? J Fam Violence. 2013;28:471-7.

15. Tong A, Sainsbury P, Craig J. Consolidated criteria for reporting qualitative research (COREQ): a 30-item checklist for interviews and focus groups. Int J Qual Health Care. 2007;19(6):349-357.

16. National Resource Council. Elder Mistreatment: Abuse, Neglect, and Exploitation in an Aging America. Washington DC: The National Academies Press; 2003. https://www.ncbi.nlm.nih.gov/books/n/nap10406/ pdf/. Accessed 12 December 2018.

17. Basile KC, Hertz MF, Back SE.Intimate Partner Violence and Sexual Violence Victimization Assessment Instruments for Use in Healthcare Settings: Version 1. Atlanta, GA: Centers for Disease Control and Prevention, National Center for Injury Prevention and Control; 2007. https://www.cdc.gov/violenceprevention/pdf/ipv/ipvandsvscreening.pdf Accessed 12 December 2018

18. Koin D. A forensic medical examination form for improved documentation and prosecution of elder abuse. J Elder Abuse Negl. 2003; 15: 109-19.

19. Rosen T, Reisig C, LoFaso VM, et al. Describing visible acute injuries: development of a comprehensive taxonomy for research and practice. Inj Prev. 2017;23(5):340-345.

20. Glaser BG, Straus AL. The Discovery of Grounded Theory: Strategies for Qualitative Research. Chicago, IL: Aldine; 1967.

21. Strauss, A. \& Corbin, J. Basics of Qualitative Research: Grounded Theory, Procedures and Techniques. Newbury Park, CA: Sage; 1998.

22. Miles MB, Huberman AM, Saldaña J. Qualitative Data Analysis: a Methods Sourcebook. 3rd ed. Thousand Oaks, CA: Sage Publishers; 2013.

23. Saldaña $\mathbf{J}$. The Coding Manual for Qualitative Researchers. 2nd ed. Thousand Oaks, CA: Sage Publications; 2012.

24. May AN, Flude GW, Duflou J, Mengersen KL, Read-Allsopp C. External injury documentation in major trauma victims is inadequate: grounds for routine photography in the emergency department? Emerg Med Australas. 2008;20:500-7.

25. Ahmad A, Lachs MS. Elder abuse and neglect: what physicians can and should do. Cleve Clin J Med. 2002;69(10):801-808.

26. Gironda MW, Nguyen AL, Mosqueda LM. Is this broken bone because of abuse? Characteristics and comorbid diagnoses in older adults with fractures. J Am Geriatr Soc. 2016;64:1651-5.

27. Murphy K, Waa S, Jaffer H, Sauter A, Chan A. A literature review of findings in physical elder abuse. Can Assoc Radiol J. 2013;64:10-4.

28. Rosen T, Bloemen EM, LoFaso VM, Clark S, Flomenbaum NE, Lachs MS. Emergency department presentations for injuries in older adults independently known to be victims of elder abuse. J Emerg Med. 2016;50:518-26.

29. Wiglesworth A, Austin R, Corona M, et al. Bruising as a marker of physical elder abuse. J Am Geriatr Soc. 2009;57:1191-6.

30. Ziminaki CE, Wiglesworth A, Austin R, Phillips LR, Mosqueda L. Injury patterns and causal mechanisms of bruising in physical elder abuse. J Forensic Nurs. 2013;9:84-91; quiz E1-2.

31. Ziminski CE, Phillips LR, Woods DL. Raising the index of suspicion for elder abuse: cognitive impairment, falls, and injury patterns in the emergency department. Geriatr Nurs. 2012;33:105-12.

32. Bryson D. Current issues: consent for clinical photography. J Vis Commun Med. 2013;36(1-2):62-3.

33. Burns K, Belton S. Clinicians and their cameras: policy, ethics and practice in an Australian tertiary hospital. Aust Health Rev. 2013;37(4):437-41.

34. Hill K. Consent, confidentiality and record keeping for the recording and usage of medical images. J Vis Commun Med. 2006;29(2):76-9.

35. Payne KF, Tahim A, Goodson AM, Delaney M, Fan K. A review of the current clinical photography guidelines in relation to smartphone publishing of medical images. J Vis Commun Med. 2012;35(4): 188-92.

36. Paryavi E, Schwartz BS, Meyer CL, Herman MJ, Abzug JM. Reliability and effectiveness of smartphone technology for the diagnosis and treatment planning of pediatric elbow trauma. J Pediatr Orthop. 2016;36(5):483-7.

37. Nagvi GA, Daly M, Dawood A, Kurkuri A, Kutty S. Smart consultation for musculoskeletal trauma: accuracy of using smart phones for fracture diagnosis. Surgeon. 2014;12(1):32-4.

38. Bullard TB, Rosenberg MS, Ladde J, Razack N, Villalobos HJ, Papa L. Digital images taken with a mobile phone can assist in the triage of neurosurgical patients to a level 1 trauma centre. J Telemed Telecare. 2013;19(2):80-3.

39. Fernandes-Taylor S, Gunter RL, Bennett KM, et al. Feasibility of implementing a patient-centered postoperative wound monitoring program using smartphone images: a pilot protocol. JMIR Res Protoc. 2017;6(2):e26.

40. Gunter R, Fernandes-Taylor S, Mahnke A, et al. Evaluating patient usability of an image-based mobile health platform for postoperative wound monitoring. JMIR Mhealth Uhealth. 2016;4(3):e133.

41. Kunde L, McMeniman E, Parker M. Clinical photography in dermatology: ethical and medico-legal considerations in the age of digital and smartphone technology. Australas J Dermatol. 2013;54(3): 192-7.

42. Capterra. The top 20 most popular ERM softward solutions. http://www. capterra.com/infographics/top-emr-software. Published January 25, 2012. Updated 2017. Accessed 12 December 2018. 